\title{
Impact of surgery in otosclerosis on the non-operated ear
}

Authors' Contribution

A-Study Design

B-Data Collection

C-Statistical Analysis

D-Manuscript Preparation

E-Literature Search

F-Funds Collection
Agnieszka Wiatr ${ }^{\mathrm{ABDE}}$, Katarzyna Job ${ }^{\mathrm{CEF}}$, Jacek Składzień ${ }^{\mathrm{ABD}}$, Maciej Wiatr ${ }^{\mathrm{BCDC}}$

Department of Otolaryngology, Jagiellonian University Medical College in Krakow, Poland; Head: prof. Jacek Składzień MD PhD

Introduction: Otosclerosis is an underlying disease of the bony labyrinth. The associated hearing loss is most often of conductive nature, in some cases the involvement of the bony part of the cochlea results in mixed hearing loss.

Aim: The aim of the analysis was to answer the question whether a surgery on one of the ears affects the state of the other ear in the course of otosclerosis.

Methods: The analysis included 140 patients hospitalized and operated on between 2010-2016. Only those patients who did not undergo a surgical operation in the middle ear due to otosclerosis prior to the study were enrolled. An audiological assessment was performed with the use of pure-tone threshold audiometry.

Results: In the group of patients with no Carhart's notch, the mean bone conduction threshold was statistically lower than before the procedure for the frequencies of $500,1000 \mathrm{~Hz}$ and statistically equal for the frequency of $2000 \mathrm{~Hz}$. The same analysis in the group of patients with Carhart's notch present in the presurgical tonal audiogram of the non-operated ear showed a statistically significant lower value of the postoperative bone conduction threshold.

Conclusions: The study confirmed the possibility of improving hearing in the non-operated ear after stapedotomy in the opposite ear by an average of $5 \mathrm{~dB}$ in the low-frequency range.

KEYWORDS: $\quad$ Carhart's notch, non-operated ear, otosclerosis, stapedotomy

\section{INTRODUCTION}

Otosclerosis is an underlying disease of the bony labyrinth. The disorder, which occurs only within the temporal bone, is characterized by progressive hearing loss and tinnitus [1-3]. The associated hearing loss is most often of conductive nature, in some cases the involvement of the bony part of the cochlea results in mixed hearing loss. Otosclerosis of the bony labyrinth without immobilization of the stirrup leads to sensorineural hearing loss. The disease has a long-term course and can lead to severe hypoacusis and even deafness [4-6]. At the initial stage of disease, hearing loss can be unilateral, later bilateral and usually asymmetrical.

\section{AIMS}

The aim of the analysis was to answer the question whether a surgery on one of the ears affects the state of the other ear in the course of otosclerosis.

\section{MATERIAL AND METHOD}

The analysis included patients hospitalized and operated on at the Clinic of Otolaryngology of the Jagiellonian University Medical College in 2010-2016.
Only those patients who did not undergo a surgical operation in the middle ear due to otosclerosis prior to the study were enrolled.

Patients who had previously undergone an ear surgery due to trauma, cancer, tympanosclerosis, chronic otitis media or had undergone a reoperation of the ear were excluded from the study group. Patients who had undergone an exploratory surgery due to a peripheral facial nerve palsy and those operated on due to a congenital anomality of the external and middle ear were also not considered.

All patients who were operated on consented to the surgery and participation in the study.

Surgery was performed on the patients by two cofosurgeons, who used the same surgical technique and had similar experience in the surgical treatment of otosclerosis. This allowed for treating the analysed patients as a homogeneous group.

The analysis included 140 patients who met the inclusion criteria chosen from a total of 172 patients operated for otosclerosis at the Clinic of Otolaryngology, Jagiellonian University Medical College.

The youngest patient was 19 years old, while the oldest was 62 years old. The average age was 39.31 years. The age structure of the studied group is presented in Fig. 1. 
Tab. I. Division of operated patients into groups.

\begin{tabular}{|c|c|c|}
\hline GROUP NO. & GROUP SIZE & SPECIFICATION OF GROUPS \\
\hline \multicolumn{3}{|c|}{ Reference group. } \\
\hline Group o & 20 & $\begin{array}{l}\text { Reference group-osseous conduction of o-20 dB, completely immobilized stirrup in the } \\
\text { oval window, no lesions in the middle ear. Stapedotomy performed. }\end{array}$ \\
\hline \multicolumn{3}{|c|}{ Subgroups: stapedotomy/stapedectomy. } \\
\hline A & 106 & Stapedotomy. \\
\hline B & 14 & Stapedectomy. \\
\hline Group $A(C+)$ & 31 & Stapedotomy. Patients with Carhart's notch in the non-operated ear before surgery. \\
\hline Group A(C-) & 95 & Stapedotomy. Patients without Carhart's notch in the non-operated ear before surgery. \\
\hline
\end{tabular}

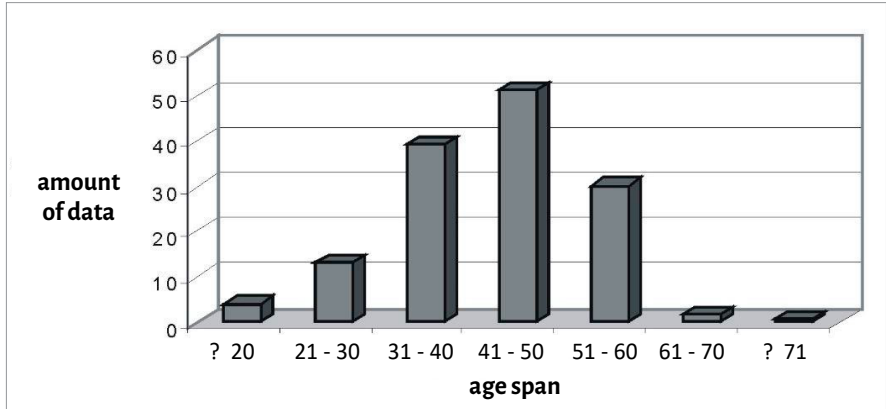

Fig. 1. Age structure of the analyzed group.

The analysed group of patients (N: 140) was divided into two subgroups depending on the type of surgery performed (subgroup A: stapedotomy, N: 126, subgroup B: stapedectomy, N: 14).

In the situation lacking the possibility for isolating a control group, which would constitute a pool of healthy people, a reference group from the A subgroup was designated, which was called group 0.

The reference group consisted of patients who, apart from complete immobilization of the stirrup base in the oval window, had no other lesions in the middle ear, and who underwent stapedotomy. An additional criterion for the inclusion of patients into the reference group was the value of the bone conduction threshold of the operated ear, which fell within the range of $0-20 \mathrm{~dB}$. The reference group consisted of 20 patients, including 16 women and 4 men. The youngest operated patient in the analysed group was 27 years old and the oldest was 46 years old. The average age in this group was 37.53 years.

The patients from subgroup A (including the reference group 0) were divided into further subgroups depending on the presence or absence of Carhart's notch in the non-operated ear $[\mathrm{A}(\mathrm{C}+)$ - Carhart's notch present in the non-operated ear, before surgery $\mathrm{N}: 31$, $\mathrm{A}(\mathrm{C}-)$ - Carhart's notch absent in the non-operated ear, before surgery N: 95]. The division of patients is presented in Tab. I.

All patients participated in an otorhinolaryngological interview and physical examination conducted in addition to a complete set of audiological examinations, including the acumetric test, tuning tests, pure-tone threshold audiometry, speech audiometry and impedance audiometry.

The patients underwent an audiological assessment with the use of pure-tone threshold audiometry taking into account:
1. the values of osseous conduction in the non-operated ear (mean bone conduction values for thresholds for pure tones in the 500, 1000, $2000 \mathrm{~Hz}$ frequency range were analysed);

2. the presence or absence of Carhart's notch in the non-operated ear.

The audiometric tests were performed at the Department and Clinic of Otolaryngology at the Jagiellonian University Medical College in a sound-proof cabin of the audiometric lab. In the cabin, the equivalent sound level corrected by the A-weighting characteristic did not exceed $25 \mathrm{dBA}$ (L Aeg = 25.1 dBA). The stimulus intensity for each tested frequency was determined with an accuracy of $5 \mathrm{~dB}$. The interior of the room was always occupied only by the examined person. Threshold values for air and bone conduction were determined by the Madsen ${ }^{\ominus}$ MIDIMATE ${ }^{\oplus}$ 622 audiometer equipped with TDK $39^{\circ}$ headphones. The audiometer met the ISO0389-1985 standard and the ISO7566-1987 standard for air conduction and bone conduction, respectively. The assessment of Polish speech comprehension was performed with an $\mathrm{AAD}^{\circ} 0^{\circ}$ audiometer and a Technics ${ }^{\circ}$ cassette tape amplifier and player. The fluctuation of the signal volume did not exceed $1.5 \mathrm{~dB}$, and the signal-to-noise ratio exceeded $63 \mathrm{~dB}$. In the examination, the speech test NLA-93 was applied. The test material consisted of 10 balanced lists, each of which contained 24 monosyllabic nouns. The test was balanced acoustically, grammatically, phonemically, semantically, energetically and structurally. It test was always carried out by the same person, with the same apparatus and the norm was established on the basis of an examination of healthy Poles. The data obtained during the research was subjected to statical analysis in cooperation with the Department of Bioinformatics and Telemedicine of the Jagiellonian University Medical College. The first test used to analyse hearing improvement was the Fisher test, also known in the relevant literature as the variance analysis test. The Fisher test measures the impact of the classifying factor on the results of the conducted study. In the work, the null hypothesis indicates that the mean values of given parameters are statistically equal. The alternative hypothesis is the inverse theorem, stating that the means are not equal. The statistical test employing the variance analysis test consisted of finding the F0 function. The null hypothesis Ho stating that all means are equal was rejected with the risk of error of $\alpha$ when F0 $>\alpha F N-k k-1$. In a study verifying nonparametric hypotheses, the test of multi-dimensional contingency tables (division tables) was used - the Chi-Square test. 
Tab. II. Change in the mean bone conduction threshold $(\triangle B C)$ in the non-operated ear after stapedotomy in the opposite ear.

\begin{tabular}{|c|c|c|c|c|c|c|c|}
\hline & AVERACE & SD & $\Delta \mathrm{BC}$ & SD & $T$ & DF & $\mathbf{P}$ \\
\hline $\mathrm{BC}^{\prime} \mathrm{O}(500 \mathrm{~Hz})$ & 22.74 & 11.41 & & & & & \\
\hline $\mathrm{BC}^{\prime} 12(500 \mathrm{~Hz})$ & 17.89 & 10.09 & 4.84 & 7.94 & 5.94 & 94 & 0.000001 \\
\hline$B^{\prime} \circ(1000 \mathrm{~Hz})$ & 24.74 & 12.06 & & & & & \\
\hline $\mathrm{BC}^{\prime} 12(1000 \mathrm{~Hz})$ & 20.58 & 11.51 & 4.16 & 8.30 & 4.88 & 94 & 0.000004 \\
\hline BC'O (2000 Hz) & 28.58 & 13.62 & & & & & \\
\hline BC'12 (2000 Hz) & 27.53 & 13.52 & 1.05 & 8.41 & 1.22 & 94 & 0.225407 \\
\hline
\end{tabular}

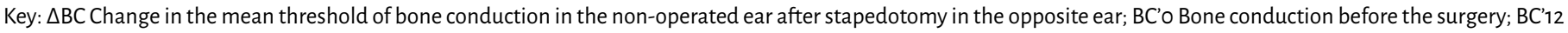
Bone conduction 12 months after the surgery.

Tab. III. Change in bone conductin (BC) in the non-operated ear after stapedotomy in the opposite ear (reference group).

\begin{tabular}{|c|c|c|c|c|c|c|c|}
\hline & AVERACE & SD & $\Delta \mathrm{BC}$ & SD & $\mathbf{T}$ & DF & $\mathbf{P}$ \\
\hline $\mathrm{BC}^{\prime} \mathrm{O}(500 \mathrm{~Hz})$ & 25.00 & 16.16 & & & & & \\
\hline $\mathrm{BC}^{\prime} 12(500 \mathrm{~Hz})$ & 20.00 & 12.80 & 5.00000 & 7.45 & 2.92 & 18 & 0.009062 \\
\hline 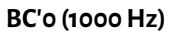 & 26.05 & 17.29 & & & & & \\
\hline$B C^{\prime} 12(1000 \mathrm{~Hz})$ & 20.53 & 13,32 & 5.52632 & 8.48 & 2.84 & 18 & 0.010857 \\
\hline BC'O (2000 Hz) & 27.37 & 20.37 & & & & & \\
\hline BC'12 (2000 Hz) & 27.37 & 18.21 & -0.00000 & 6.24 & -0.000000 & 18 & 1.000000 \\
\hline
\end{tabular}

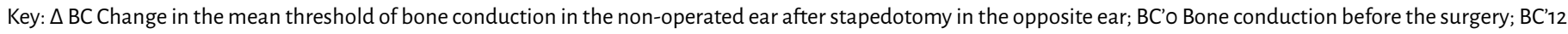
Bone conduction 12 months after the surgery.

Tab. IV. Change in bone conduction $(\triangle B C)$ in the non-operated ear after stapedectomy in the opposite ear.

\begin{tabular}{|c|c|c|c|c|c|c|c|}
\hline & AVERAGE & SD & $\Delta \mathrm{BC}$ & SD & $T$ & DF & $\mathbf{P}$ \\
\hline $\mathrm{BC}^{\prime} \mathrm{O}(500 \mathrm{~Hz})$ & 25.38 & 6.91 & & & & & \\
\hline $\mathrm{BC}^{\prime} 12(500 \mathrm{~Hz})$ & 22.30 & 8.06 & 3.07 & 7.51 & 1.48 & 12 & 0.17 \\
\hline BC'O (1000 Hz) & 27.69 & 9.92 & & & & & \\
\hline $\mathrm{BC}^{\prime} 12(1000 \mathrm{~Hz})$ & 23.46 & 8.75 & 4.23 & 8.62 & 1.77 & 12 & 0.10 \\
\hline BC'O $(2000 \mathrm{~Hz})$ & 33.46 & 13.90 & & & & & \\
\hline 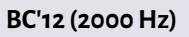 & 32.31 & 11.11 & 1.15 & 8.45 & 0.49 & 12 & 0.63 \\
\hline
\end{tabular}

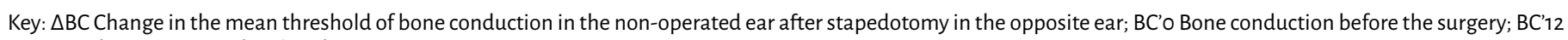
Bone conduction 12 months after the surgery.

This test is used for non-measurable variables of a qualitative nature. In this test, Pearson's chi-squared test was used as the method of calculating $\mathrm{c} 2$, which was confirmed by the Chi-Square goodness of fit test. This method allows to analyse whether the data representing variables contained in a division table provide sufficient proof of a correlation between these variables. The correlation significance was shown Cramér's V statistic, whose value always falls between 0 and 1 . The greater the correlation between the variables, the closer the value of the coefficient is to 1 . This paper assumes a significance level of $5 \%$ (alpha $=0.05)$, which means that the risk of rejecting a hypothesis in the case of its validity is $5 \%$. Statistically significant results were reported for $\mathrm{p}<0.05$.

\section{RESULTS}

Changes in the mean threshold of bone conduction in the nonoperated ear were observed. The aim of the analysis was to answer the question whether a surgery on one of the ears affects the state of the other ear. In the first place, the study was conducted on the group of patients who had undergone stapedotomy and the following results were obtained, $\mathrm{p}<0.05$ (Tab. II.).

The same analysis in the reference group allowed to obtain the following results (Tab. III.).

The statistical analysis of the results gives grounds to assume that the average threshold value of bone conduction in the non-operated ear is statistically lower than before the surgery for frequencies of 500, $1000 \mathrm{~Hz}$ and statistically equal for the frequency of $2000 \mathrm{~Hz}$. This confirms the possibilities of improving hearing in the non-operated ear after stapedotomy in the opposite ear by an average of $5 \mathrm{~dB}$ in the low-frequency range.

A similar analysis in the group of patients who had undergone stapedectomy allowed to obtain the following results after a 12-month observation (Tab. IV.). 
Tab. V. Change in bone conduction $(\triangle B C)$ in the non-operated ear, without Carhart's notch $A(C-)$, after stapedotomy in the opposite ear.

\begin{tabular}{|c|c|c|c|c|c|c|c|}
\hline & AVERAGE & SD & $\Delta \mathrm{BC}$ & SD & $T$ & DF & $\mathbf{P}$ \\
\hline $\mathrm{BC}^{\prime} \mathrm{O}(500 \mathrm{~Hz})$ & 22.53 & 11.34 & & & & & \\
\hline $\mathrm{BC}^{\prime} 12(500 \mathrm{~Hz})$ & 18.37 & 10.68 & 4.16 & 7.35 & 5.51 & 94 & 0.000001 \\
\hline $\mathrm{BC}^{\prime} 0(1000 \mathrm{~Hz})$ & 25.37 & 12.93 & & & & & \\
\hline $\mathrm{BC}^{\prime} 12(1000 \mathrm{~Hz})$ & 21.00 & 11.93 & 4.23 & 4.37 & 8,23 & 94 & 0.000001 \\
\hline$B^{\prime} \circ(2000 \mathrm{~Hz})$ & 26.47 & 13.83 & & & & & \\
\hline BC'12 (2000 Hz) & 26.37 & 13.59 & 0.10 & 7.99 & 0.13 & 94 & 0.898084 \\
\hline
\end{tabular}

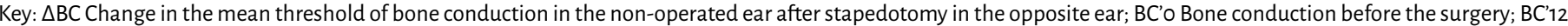
Bone conduction 12 months after the surgery.

Tab. VI. Change in bone conduction $(\triangle B C)$ in the non-operated ear with Carhart's notch $A(C+)$, after stapedotomy in the opposite ear.

\begin{tabular}{|c|c|c|c|c|c|c|c|}
\hline & AVERAGE & SD & $\Delta \mathrm{BC}$ & SD & $\mathbf{T}$ & DF & $\mathbf{P}$ \\
\hline $\mathrm{BC}^{\prime} \mathrm{O}(500 \mathrm{~Hz})$ & 25.78 & 13.08 & & & & & \\
\hline $\mathrm{BC}^{\prime} 12(500 \mathrm{~Hz})$ & 19.53 & 9.53 & 6.25 & 8.89 & 3.97 & 31 & 0.000389 \\
\hline 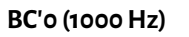 & 24.84 & 12.21 & & & & & \\
\hline $\mathrm{BC}^{\prime} 12(1000 \mathrm{~Hz})$ & 20.47 & 10.27 & 4.37 & 8.68 & 2.85 & 31 & 0.007703 \\
\hline BC'O (2000 Hz) & 36.093 & 15.38 & & & & & \\
\hline $\mathrm{BC}^{\prime} 12(2000 \mathrm{~Hz})$ & 32.81 & 14.53 & 3.28 & 7.99 & 2.32 & 31 & 0.026958 \\
\hline
\end{tabular}

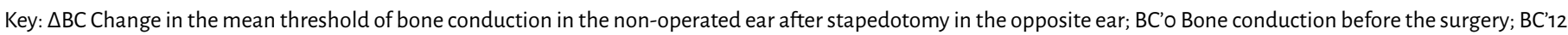
Bone conduction 12 months after the surgery.

The statistical analysis of the results gives grounds to conclude that the mean bone conduction thresholds are statistically equal (at $\mathrm{p}<0.05$ ). This means that after stapedectomy, there was no improvement in the analysed parameter in the opposite ear at any frequency.

The subsequent part of the study aimed to answer the question whether the improvement of the bone conduction value in the non-operated ear after stapedotomy in the opposite ear is dependent on the presence of Carhart's notch in the non-operated ear.

The following results were obtained in the group of patients who had no Carhart's notch in the non-operated ear $(\mathrm{N}=95$ people $)$, after stapedotomy in the opposite ear (Tab. V.).

In the group of patients with Carhart's notch in the non-operated ear ( $\mathrm{N}=32$ people), the following results were obtained after stapedotomy in the opposite ear, p $<0.05$ (Tab. VI.).

In the group of patients with no Carhart's notch, the mean threshold of bone conduction was statistically lower than before the procedure for frequencies of $500,1000 \mathrm{~Hz}$ and statistically equal for the frequency of $2000 \mathrm{~Hz}$. The same analysis in the group of patients with Carhart's notch present in the pre-surgical tonal audiogram performed on the non-operated ear showed a statistically significant lower value of the post-operative bone conduction threshold than before the procedure for frequencies of 500, 1000, $2000 \mathrm{~Hz}$.

Due to the statistically small group of patients who had undergone stapedectomy, the impact of Carhart's notch on the nonoperated ear after surgery of the opposite ear was not analyzed.

\section{DISCUSSION}

Many authors of publications in the field of stapes surgery are unanimous regarding the improvement of bone conduction in the early period after uncomplicated surgery for otosclerosis. Stapedotomy without any complications allows to improve the bone conduction threshold in the operated ear. This is done by restoring the three bone conduction pathways described as early as in 1968 by Tondorf (directly from the bones to the cochlea, from the bones to the middle ear and the cochlea, from the bones to the external auditory canal, then to the middle ear and to the cochlea). Stapedotomy increases the contribution of the middle ear to bone conduction (the Carhart effect) and reduces the size of the conductive component. Controversies and discussions emerge in the analysis of bone conduction changes in the distant period after surgery in the operated ear. Even greater discussions and discrepancies in opinions appear regarding early and late post-operative changes in the non-operated ear after surgery on the opposite ear. The impact of surgery in otosclerosis on the condition of the other, non-operated ear during early observation was already discussed in the scientific works of Prof. J. Miodoński. While supporting the observations of other authors (Güttich, Tulio, Rozenzweig, Kraus, Kobrak), Miodoński concurrently points to the fact that, unlike in the case of the right and left vestibular organs, where there is a close interdependence, correlations are also to be found between ears. In the 1960s, Lempart, Shambaugh, Wood, and Zangenmeister spoke critically about this theory. The results presented in 1995 by Pirodda et al. of the analysis performed on a large group of 200 patients operated for otosclerosis during a follow-up period of $13.4+/-5.3$ years after surgery did not show any deterioration of bone conduction in the operated and non-operated ears $[7,8]$. 
Similar observations were presented by other authors, who found a deterioration of bone conduction in both the operated and nonoperated ears during observations which took place a long time after the surgery. A deterioration of bone conduction in a distant meta-analysis in the non-operated ear was confirmed by Smyth et al., at the same time showing no deterioration in the operated ear [9-12]. It is difficult to clearly explain the improvement in bone conduction in the non-operated ear after stapedotomy performed on the opposite ear for frequencies of 500 and 1000 $\mathrm{Hz}$ during a 12-month follow-up, showcased by our study. This may be related to the appearance and transfer of Carhart's effect to the non-operated ear, consisting in a reduction in the size of the conductive component of hearing loss and an increase in the contribution of the middle ear in bone conduction. Due to the complex course of the condition and the progressive nature of changes in otosclerosis, the above observations require a broader analysis, as well as an observation that would exceed one year.

\section{CONCLUSIONS}

1. The observations are attractive from the point of view of qualifying patients for surgical treatment and the subsequent decision regarding surgery in the other, previously non-operated ear;

2. Due to the complex course of the condition and the progressive nature of changes in otosclerosis, the above observations require a broader analysis, as well as an observation of over one year.

\section{FUNDING}

This research was supported by statutory funds of the Department of Otolaryngology of the Jagiellonian University, Krakow, Poland.

\section{REFERENCES}

1. Eshraghi A.A., Telischi F.F.: Otosclerosis and Stapes Surgery. Otolaryngol Clin North Am., 2018; 51(2): 17-19.

2. Hall A.C., Mandavia R., Selvadurai D.: Total endoscopic stapes surgery: Systematic review and pooled analysis of audiological outcomes. Laryngoscope., 2020; 130(5): 1282-1286

3. Eshraghi A.A., Ila K., Ocak E., Telischi F.F.: Advanced Otosclerosis: Stapes Surgery or Cochlear Implantation? Otolaryngol Clin North Am., 2018; 51(2): 429-440.

4. Sobolewska A., Clarós P.: Surgical treatment in children with otosclerosis and congenital stapes fixation: our experience and outcome. Otolaryngol Pol, 2019; 73(2):23-28.

5. Manna S., Kaul V.F., Gray M.L., Wanna G.B.: Endoscopic Versus Microscopic Middle Ear Surgery: A Meta-analysis of Outcomes Following Tympanoplasty and Stapes Surgery.Otol Neurotol., 2019; 40(8): 983-993.

6. Dwyer-Hemmings L., Manjaly J.G., Nash R., Mukherjee A., Lavy J.A.: Stapes Surgery for Profound Hearing Loss Secondary to Otosclerosis.Ear Nose Throat J., 2019; 98(5): 273-278.
7. Alicandri-Ciufelli M., Molinari G., Rosa M.S., Monzani D., Presutti L.: Gusher in stapes surgery: a systematic review. Eur Arch Otorhinolaryngol., 2019; 276(9): 2363-2376.

8. Nazarian R., McElveen J.T. Jr, Eshraghi A.A.: History of Otosclerosis and Stapes Surgery. Otolaryngol Clin North Am., 2018; 51(2): 275-290.

9. Soudry E., Sulkes J., Attias J., Nageris B.I.: Bone conduction in otosclerosis-operated versus non-operated ears.J Basic Clin Physiol Pharmacol., 2007; 18(3): 189-199.

10. Quesnel A.M., Ishai R., McKenna M.J.: Otosclerosis: Temporal Bone Pathology. Otolaryngol Clin North Am., 2018; 51(2): 291-303.

11. Amadei E.M., Cola C.: Revision stapes surgery after stapedotomy: A retrospective evaluation of 75 cases. Ear Nose Throat J., 2018; 97(6): E1-E4.

12. Yeh C.F., Wang M.C., Chu C.H., Shiao A.S.: Predictors of hearing outcomes after stapes surgery in otosclerosis. Acta Otolaryngol., 2019; 139(12): 1058-1062.

Word count: 2504 Tables: 6 Figures: 1 References: 12

Access the article online: DOI: 10.5604/01.3001.0014.5675 Table of content: https://otolaryngologypl.com/issue/13708

Some right reserved: Polish Society of Otorhinolaryngologists Head and Neck Surgeons. Published by Index Copernicus Sp. z o.o.

Competing interests: The authors declare that they have no competing interests.

The content of the journal „Polish Society of Otorhinolaryngologists Head and Neck Surgeons” is circulated on the basis of the Open Access which means free and

limitless access to scientific data.

This This material is available under the Creative Commons - Attribution-NonCommercial 4.0 International (CC BY-NC 4.0). The full terms of this license are available on: https://creativecommons.org/licenses/by-nc/4.0/legalcodee

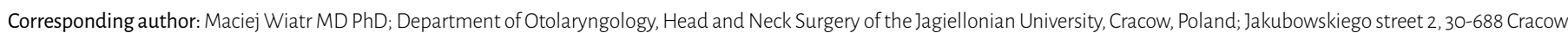

Poland; Phone: +4812400 2750; E-mail: maciej.wiatr@uj.edu.pl

Cite this article as: Wiatr A., Job K., Skladzien ]., Wiatr M.: Impact of surgery in otosclerosis on the non-operated ear; Otolaryngol Pol, 2021: 75 (2): 1-6 
